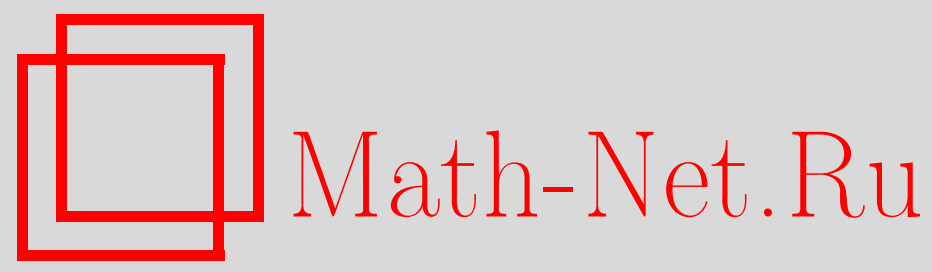

В. Л. Куракин, Функция переноса в первый разряд в кольце Галуа, Дискрет. матем., 2012, том 24, выпуск 2, 21-36

DOI: https://doi.org/10.4213/dm1181

Использование Общероссийского математического портала Math-Net.Ru подразумевает, что вы прочитали и согласны с пользовательским соглашением http://www . mathnet.ru/rus/agreement

Параметры загрузки:

IP: 35.173 .219 .12

26 апреля 2023 г., 16:24:55 
УДК 519.7

\title{
Функция переноса в первый разряд в кольце Галуа
}

\author{
() 2012 г. В. Л. Куракин
}

В кольце Галуа $R=G R\left(q^{n}, p^{n}\right)$ вводится общее понятие координатного множества по аналогии с координатным множеством $\{0, \ldots, p-1\}$ примарного кольца вычетов $\mathbf{Z}_{p^{n}}$. Любое такое множество $B$ позволяет однозначно представить каждый элемент $a \in R$ в виде

$$
a=\sum_{s \in\{0, \ldots, n-1\}} b_{s} p^{s}, \quad b_{s} \in B,
$$

и каждую функцию $U: R^{k} \rightarrow R$ в виде функции $F: B^{m} \rightarrow R, m=k n$. Последняя, в свою очередь, представляется в виде

$$
F\left(b_{1}, \ldots, b_{m}\right)=\sum_{s \in\{0, \ldots, n-1\}} F_{s}\left(b_{1}, \ldots, b_{m}\right) p^{s},
$$

где $F_{s}: B^{m} \rightarrow B-$ функция, называемая функцией переноса функции $U$ в $s$-й разряд. Каждая из этих функций представляется многочленом над полем $G F(q)$. В данной работе изучаются свойства многочлена $F_{1}$ в зависимости от способа выбора координатного множества $B$. Наиболее подробно рассматривается случай, когда $U-$ аффинная функция $R=\mathbf{Z}_{p^{n}}$ и $B=\{0, \ldots, p-1\}$. При этом обнаруживается интересная зависимость вида функции переноса от свойства числа $p$ быть регулярным.

\section{1. Координатные множества колец Галуа}

Кольцом Галуа называется конечное коммутативное локальное кольцо $R$, максимальный идеал которого равен $p R$ для некоторого простого числа $p$ (см. [7-11]). Кольцо Галуа однозначно, с точностью до изоморфизма, определяется своей характеристикой $p^{n}$ и числом элементов $q^{n}$, где $q=p^{r}$, и обозначается $G R\left(q^{n}, p^{n}\right)$. Примерами колей Галуа являются примарные кольца вычетов $\mathbf{Z}_{p^{n}}=G R\left(p^{n}, p^{n}\right)$ и конечные поля $G F(q)=G R(q, p)$.

Множество обратимых элементов кольца $R$ имеет вид $R^{*}=R \backslash p R$, и поле вычетов $\bar{R}=R / p R$ является полем из $q$ элементов: $\bar{R}=G F(q)$. Образ элемента $a \in R$ при действии естественного эпиморфизма $R \rightarrow \bar{R}$ будем обозначать через $\bar{a}$, и образ многочлена $F(x) \in R[x]$ при действии естественного эпиморфизма $R[x] \rightarrow \bar{R}[x]$ будем обозначать $\bar{F}(x)$. Будем писать, что $a \equiv b\left(\bmod p^{s}\right)$, если $a-b \in p^{s} R$. Очевидно, что $a \equiv b(\bmod p)$ тогда и только тогда, когда $\bar{a}=\bar{b}$. Кольцо Галуа $R=G R\left(q^{n}, p^{n}\right), q=p^{r}$, изоморфно факторкольцу $\mathbf{Z}_{p^{n}}[x] / G(x)$, где $G(x)$ - произвольный унитарный многочлен степени $r$ над $\mathbf{Z}_{p^{n}}$ такой, что многочлен $\bar{G}(x)$ неприводим над полем $\mathbf{Z}_{p^{n}} / p \mathbf{Z}_{p^{n}}=G F(p)$. 
Известно, что каждый элемент $c$ кольца $\mathbf{Z}_{p^{n}}$ может быть однозначно представлен в виде суммы

$$
c=c_{0}+p c_{1}+\ldots+p^{n-1} c_{n-1}, \quad 0 \leqslant c_{s} \leqslant p-1, \quad s \in\{0,1, \ldots, n-1\} .
$$

Это разложение называется $p$-ичным разложением элемента $c$. Элементы произвольного кольца Галуа $R$ имеют аналогичное разложение, в котором множество $\{0,1, \ldots, p-1\}$ заменяется координатным множеством кольца Галуа.

Подмножество $B \subset R$ называется координатным множеством кольца Галуа $R$, если $0 \in B$ и элементы множества $B$ образуют полную систему вычетов по модулю идеала $p R$, то есть, $\bar{B}=\bar{R}$. Аналогично утверждению 1 в [8], доказывается, что каждый элемент $c \in R$ однозначно представляется в виде

$$
c=c_{0}+p c_{1}+\ldots+p^{n-1} c_{n-1}, \quad c_{s} \in B, \quad s \in\{0,1, \ldots, n-1\},
$$

называемом разложением элемента $c$ в координатном множестве $B$. Отображение

$$
\gamma_{s}^{B}: R \rightarrow B, \quad \gamma_{s}^{B}(c)=c_{s}, \quad s \in\{0,1, \ldots, n-1\},
$$

называется $s$-й координатной функцией множества $B$ в координатном множестве $B$, а элементы $c_{s}=\gamma_{c}^{B}(c) \in B$ называются координатами элемента $c$ в координатном множестве $B$.

Введем на множестве $B$ операции

$$
a \oplus b=\gamma_{0}^{B}(a+b), \quad a \otimes b=\gamma_{0}^{B}(a b), \quad a, b \in B .
$$

Тогда $(B, \oplus, \otimes)=G F(q)$ - поле, изоморфное $\bar{R}$, причем изоморфизм задается соотношениями

$$
\varphi: B \rightarrow \bar{R}, \quad \varphi(\alpha)=\bar{\alpha}, \quad \alpha \in B
$$

Двумя важными примерами координатных множеств являются $p$-адическое и $p$-ичное координатные множества. В силу леммы 3 в [8], множество

$$
\Gamma(R)=\left\{c \in R: c^{q}=c\right\}
$$

является координатным множеством кольца Галуа $R$, которое называется $p$-адическим координатным множеством. Из определения следует, что множество $\Gamma(R)$ замкнуто относительно умножения. Поэтому операция умножения $\otimes$ на $\Gamma(R)$, определенная в (2), совпадает с операцией умножения в кольце $R$. Координатные функции в $p$-адическом координатном множестве обозначаются через $\gamma_{s}$, то есть

$$
\gamma_{s}^{\Gamma(R)}=\gamma_{s}
$$

Если $R=\mathbf{Z}_{p^{n}}$, то множество

$$
B_{p}=\{0,1,2, \ldots, p-1\} \subset \mathbf{Z}_{p^{n}}
$$

называется $p$-ичным координатным множеством кольца $\mathbf{Z}_{p^{n}}$. Разложение элемента в $p$-ичном координатном множестве совпадает с $p$-ичным разложением 1 .

Пусть $U: R^{k} \rightarrow R-$ произвольное отображение и $\vec{a}=\left(a_{1}, \ldots, a_{k}\right) \in R^{k}$. Тогда элемент $U\left(a_{1}, \ldots, a_{k}\right) \in R$ представляется в виде

$$
U(\vec{a})=U_{0}(\vec{a})+p U_{1}(\vec{a})+\ldots+p^{n-1} U_{n-1}(\vec{a}), \quad U_{s}(\vec{a}) \in B, \quad s \in\{0,1, \ldots, n-1\} .
$$


Отображение $U_{s}=\gamma_{s}^{B}(U): R^{k} \rightarrow B$ называется $s$-й координатной функцией отображения $U$ в координатном множестве $B$. Отображение $F_{s}: B^{k} \rightarrow B, F_{s}=U \mid B^{k}$, являющееся ограничением отображения $U_{s}: R^{k} \rightarrow B$ на множество $B^{k}$, будем называть функцией переноса в $s$-й разряд для отображения $U$ в координатном множестве $B$. Таким образом, для любых $b_{1}, \ldots, b_{r} \in B$

$$
U\left(b_{1}, \ldots, b_{k}\right)=F\left(b_{1}, \ldots, b_{k}\right)=F_{0}\left(b_{1}, \ldots, b_{k}\right)+\ldots+p^{n-1} F_{n-1}\left(b_{1}, \ldots, b_{k}\right),
$$

где $F_{s}\left(b_{1}, \ldots, b_{k}\right) \in B_{s}, s \in\{0,1, \ldots, n-1\}$.

Например, пусть $B$ есть $p$-ичное координатное множество кольца $\mathbf{Z}_{p^{n}}, F(a, b)=a+b$ - сумма элементов кольца. Тогда функции переноса

$$
F_{s}(a, b)=\gamma_{s}^{B}(a+b)
$$

задаются соотношениями

$$
\begin{aligned}
& \gamma_{0}^{B}(a+b)=a \oplus b, \\
& \gamma_{1}^{B}(a+b)= \begin{cases}0, & \text { если } a+b<p, \\
1, & \text { если } a+b \geqslant p,\end{cases} \\
& \gamma_{s}^{B}(a+b)=0, \quad s \geqslant 2 .
\end{aligned}
$$

Функция переноса в первый разряд при сложении изучалась в [4] (см. лемму 2.2).

Отметим, что изучение координатных функций отображения $U$ в определенном смысле сводится к изучению функций переноса. Именно, пусть $U: R^{k} \rightarrow R$ и $\left(a_{1} \ldots, a_{k}\right) \in R^{k}$. Каждый элемент $a_{i} \in R$ запишем в виде

$$
a_{i}=a_{i 0}+p a_{i 1}+\ldots+p^{n-1} a_{i, n-1},
$$

где $a_{i j} \in B$. Тогда

$$
U\left(a_{1}, \ldots, a_{k}\right)=F\left(a_{10}, \ldots, a_{1, n-1} \ldots, a_{k 0}, \ldots, a_{k, n-1}\right),
$$

и $U$ можно рассматривать как отображение $F: B^{k n} \rightarrow R$. В таком случае координатную функцию $U_{s}\left(a_{1}, \ldots, a_{k}\right)$ можно рассматривать как функцию переноса $F_{s}\left(a_{10}, \ldots, a_{k, n-1}\right)$.

Задача нахождения координатных функций и функций переноса возникает в ряде приложений, например, при построении псевдослучайных последовательностей из координатных последовательностей линейных рекуррент над кольцами Галуа [9, 4] и при исследовании нелинейных кодов, получаемых из линейных кодов над кольцами Галуа [8]. В данной работе найдена функция переноса в первый разряд $F_{1}=\gamma_{1}^{B}(F)$ для произвольного отображения $F$ в произвольном координатном множестве $B$ кольца Галуа $R$.

\section{2. Интерполяционный и характеристический многочлены координатного множества}

Пусть $R=G R\left(q^{n}, p^{n}\right)$ - кольцо Галуа и $\Gamma(R)=\left\{\beta_{0}, \beta_{1}, \ldots, \beta_{q-1}\right\}$ - его $p$-адическое координатное множество. Если $B=\left\{b_{0}, b_{1}, \ldots, b_{q-1}\right\}-$ произвольное координатное множество кольца $R$, то всегда будем предполагать, что его элементы занумерованы в таком порядке, что

$$
b_{0}=0, \quad b_{t}=\beta_{t}, \quad 0 \leqslant t \leqslant q-1 .
$$


Предложение 1. Для любого подмножества $B=\left\{b_{0}, b_{1}, \ldots, b_{q-1}\right\} \subset R$, состоящего из $q$ элементов, существует единственный многочлен

$$
\Psi_{B}(x)=\psi_{q-1} x^{q-1}+\ldots+\psi_{1} x+\psi_{0} \in R[x]
$$

такой, что

$$
\Psi_{B}\left(\beta_{t}\right)=b_{t}, \quad 0 \leqslant t \leqslant q-1 .
$$

При этом

$$
\Psi_{B}(x)=-b_{0}\left(x^{q-1}-e\right)+\frac{x}{q-1} \sum_{t=1}^{q-1} b_{t}\left(x^{q-2}+x^{q-3} \beta_{t}+\ldots+x \beta_{t}^{q-3}+\beta_{t}^{q-2}\right) .
$$

Доказательство. В случае $R=\mathbf{Z}_{p^{n}}$ это утверждение доказано в [5]. В случае произвольного кольца Галуа $R$ доказательство аналогично. А именно, коэффициенты многочлена $\Psi_{B}(x)$ являются решениями системы линейных уравнений над кольцом $R$

$$
\left(\begin{array}{cccc}
e & 0 & \ldots & 0 \\
e & \beta_{1} & \ldots & \beta_{1}^{q-1} \\
\vdots & \vdots & & \vdots \\
e & \beta_{q-1} & \ldots & \beta_{q-1}^{q-1}
\end{array}\right)=\left(\begin{array}{c}
\psi_{0} \\
\psi_{1} \\
\vdots \\
\psi_{q-1}
\end{array}\right)=\left(\begin{array}{c}
b_{0} \\
b_{1} \\
\vdots \\
b+q-1
\end{array}\right)
$$

Определитель главной матрицы этой системы есть определитель Вандермонда, поэтому главная матрица системы обратима и система имеет единственное решение. Следовательно, многочлен $\Psi_{B}(x)$ существует и определен однозначно. Пользуясь соотношениями

$$
\begin{aligned}
x^{q-2}+x^{q-3} \beta_{t}+\ldots+x \beta_{t}^{q-3}+\beta_{t}^{q-2} & =\frac{x^{q-1}-\beta_{t}^{q-1}}{x-\beta_{t}}=\frac{x^{q-1}-e}{x-\beta_{t}} \\
& =\left(x-\beta_{1}\right) \cdots\left(x-\beta_{t-1}\right)\left(x-\beta_{t+1}\right) \cdots\left(x-\beta_{q-1}\right),
\end{aligned}
$$

где $0 \leqslant t \leqslant q-1$, легко убедиться в том, что многочлен в правой части соотношения (5) удовлетворяет условиям (4). Это доказывает (5).

Следствие 1. Если $B$ - координатное множество кольца $R$, причем выполняется соотношение (3), то

$$
\Psi_{B}(0)=0, \quad \Psi_{B}(x) \equiv x \quad(\bmod p) .
$$

Равенство $\Psi_{B}(x)=x$ равносильно тому, что $B=\Gamma(R)$.

В условиях предложения 1 и следствия 1 многочлен $\Psi_{B}(x)$ будем называть интерполяционным многочленом (координатного) множества $B$. Следующий результат показывает, как с помощью интерполяционного многочлена можно вычислить нулевую координату элемента $a \in R$ в координатном множестве $B$.

Теорема 1. Для любого элемента $a \in R$ выполняется равенство

$$
\gamma_{0}^{B}(a)=\Psi_{B}\left(a^{q^{n-1}}\right) .
$$


Доказательство. Пусть $\gamma_{0}(a)=\beta_{t} \in \Gamma(R)$, где $t \in\{0,1, \ldots, q-1\}$. В силу (3),

$$
\gamma_{0}^{B}(a)=b_{t} .
$$

Согласно лемме 4 в [8],

$$
\gamma_{0}(a)=a^{q^{n-1}}
$$

Теперь, согласно предложению 1,

$$
\gamma_{0}^{B}(a)=b_{t}=\Psi_{B}\left(\beta_{t}\right)=\Psi_{B}\left(\gamma_{0}(a)\right)=\Psi_{B}\left(a^{q^{n-1}}\right),
$$

что и требовалось доказать.

Как следствие, получаем формулу для нулевой координатной функции произвольного отображения.

Следствие 2. Если $F: R^{k} \rightarrow R-$ произвольное отображение, то

$$
\gamma_{0}^{B}\left(F\left(a_{1}, \ldots, a_{k}\right)\right)=\Psi_{B}\left(F\left(a_{1}, \ldots, a_{k}\right)^{q^{n-1}}\right) .
$$

Пусть $B=\left\{b_{0}, b_{1}, \ldots, b_{q-1}\right\} \in R$ - подмножество кольца $R$, состоящее из $q$ элементов. Многочлен

$$
\chi_{B}(x)=x\left(x-b_{1}\right) \cdots\left(x-b_{q-1}\right)=x^{q}+\chi_{q-1} x^{q-1}+\ldots+\chi_{1} x
$$

над кольцом $R$ будем называть характеристическим многочленом множества $B$. Если $B$ - координатное множество, то, очевидно,

$$
\chi_{B}(x) \equiv x^{q}-x \quad(\bmod p),
$$

и, по следствию 1,

$$
\Psi_{B}(x) \equiv x \quad(\bmod p) .
$$

Обозначим через $\Psi_{B}^{*}(x)$ и $\chi_{B}^{*}(x)$ многочлены степеней не выше $q-1$ над кольцом $R$, удовлетворяющие соотношениям

$$
\Psi_{B}(x)=x+p \Psi_{B}^{*}(x), \quad \chi_{B}(x)=x^{q}-x+p \chi_{B}^{*}(x) .
$$

Между интерполяционными и характеристическими многочленами имеется следующая связь.

Предложение 2. Если $B$ - координатное множество кольйа $R$, то

$$
\Psi_{B}^{*} \equiv \chi_{B}^{*}(x) \quad(\bmod p) .
$$

Доказательство. По предложению 1,

$$
b_{t}=\Psi_{B}\left(\beta_{t}\right)=\beta_{t}+p \Psi_{B}^{*}\left(\beta_{t}\right), \quad 0 \leqslant t \leqslant q-1 .
$$

С другой стороны,

$$
b_{t}=b_{t}+\chi_{B}\left(b_{t}\right)=b_{t}+b_{t}^{q}-b_{t}+p \chi_{B}^{*}\left(b_{t}\right)=b_{t}^{q}+p \chi_{B}^{*}\left(b_{t}\right) .
$$


В силу леммы 4 из [8],

$$
b_{t}^{q} \equiv \beta_{t} \quad\left(\bmod p^{2}\right)
$$

Отсюда

$$
\Psi_{B}^{*} \equiv \chi_{B}^{*}\left(b_{t}\right) \quad(\bmod p), \quad 0 \leqslant t \leqslant q-1 .
$$

С учетом (3) это означает, что

$$
\bar{\Psi}_{B}^{*}(\bar{a})=\bar{\chi}_{B}^{*}(\bar{a})
$$

для любого $\bar{a} \in \bar{R}$. Так как степени многочленов $\Psi_{B}^{*}(x)$ и $\chi_{B}^{*}(x)$ не превосходят $q-1$, то

$$
\bar{\Psi}_{B}^{*}(x)=\bar{\chi}_{B}^{*}(x) .
$$

Следствие 3. Если $B$ - координатное множество кольц̧а $R$, то коэффициентьл многочленов $\Psi_{B}(x)$ и $\chi_{B}(x)$ с номерами $l \in\{2, \ldots, q-1\}$ сравнимы по модулю $p^{2}$ :

$$
\psi_{l} \equiv \chi_{l} \quad\left(\bmod p^{2}\right), \quad 2 \leqslant l \leqslant q-1 .
$$

\section{3. Функция переноса в первый разряд}

Пусть $B$ - координатное множество кольца Галуа $R=G R\left(q^{n}, p^{n}\right), F: B^{k} \rightarrow R-$ некоторое отображение и

$$
F_{s}=\gamma_{s}^{B}(F): B^{k} \rightarrow B, \quad 0 \leqslant s \leqslant n-1,
$$

- функция переноса в $s$-й разряд. Заметим, что для любой функции $G: R^{k} \rightarrow R$ такой, что

$$
F_{S}(\vec{a}) \equiv G(\vec{a}) \quad(\bmod p), \quad \vec{a} \in B^{k},
$$

в силу теоремы 1 выполняется равенство

$$
F_{S}=\Psi_{B}\left(G^{q^{n-1}}\right)
$$

Поэтому достаточно вычислить функцию $F_{S}$ по модулю $p$, другими словами, достаточно описать функцию $\bar{F}_{s}: B^{k} \rightarrow \bar{R}$.

Предложение 3. Произвольное отображение $F: B^{k} \rightarrow R$ является полиномиальным, то есть, существует многочлен $H\left(x_{1}, \ldots, x_{k}\right)$ над кольциом $R$ такой, что

$$
F(\vec{a})=H(\vec{a})
$$

для любого $\vec{a}=\left(a_{1}, \ldots, a_{k}\right) \in B^{k}$.

Доказательство. Для элемента $a \in B$ рассмотрим многочлен

$$
I_{a}(x)=\prod_{b \in B \backslash a} \frac{x-b}{a-b}
$$

над кольцом $R$. Так как $B-$ координатное множество, то элементы $a-b$, где $b \in B \backslash a$, обратимы, и многочлен $I_{a}(x)$ определен корректно. Очевидно, что

$$
I_{a}(c)= \begin{cases}1, & c=a, \\ 0, & c \in B \backslash a .\end{cases}
$$


Тогда для многочлена $H$, заданного интерполяционной формулой

$$
H\left(x_{1}, \ldots, x_{k}\right)=\sum_{\left(a_{1}, \ldots, a_{k}\right) \in B^{k}} I_{a_{1}}\left(x_{1}\right) \cdots I\left(a_{k}\right) F\left(a_{1}, \ldots, a_{k}\right),
$$

выполняется условие $F(\vec{a})=H(\vec{a}), \vec{a} \in B^{k}$.

С учетом предложения 3 будем считать, что отображение $F: B^{k} \rightarrow R$ задано многочленом $F=F\left(x_{1}, \ldots, x_{k}\right) \in R\left[x_{1}, \ldots, x_{k}\right]$. Тогда

$$
F\left(x_{1}, \ldots, x_{k}\right)^{q} \equiv F\left(x_{1}^{q}, \ldots, x_{k}^{q}\right) \quad(\bmod p) .
$$

Обозначим через $F^{*}\left(x_{1}, \ldots, x_{k}\right)$ многочлен над кольцом $R$, удовлетворяющий соотношению

$$
F\left(x_{1}, \ldots, x_{k}\right)^{q}=F\left(x_{1}^{q}, \ldots, x_{k}^{q}\right)+p F^{*}\left(x_{1}, \ldots, x_{k}\right) .
$$

Через $\frac{\partial F}{\partial x_{i}}$ будем обозначать формальную частную производную многочлена $F$ по переменной $x_{i}, 1 \leqslant i \leqslant k$. Эти производные являются многочленами из $R\left[x_{1}, \ldots, x_{k}\right]$. Через $\frac{\partial F}{\partial x_{i}}\left(a_{1}, \ldots, a_{k}\right)$ будем обозначать значение частной производной в точке $\left(a_{1}, \ldots, a_{K}\right) \in R^{k}$.

Теорема 2. Пусть $B-$ координатное множество кольйа Галуа $R=G R\left(q^{n}, p^{n}\right) u$

$$
\Psi_{B}(x)=x+p \Psi_{B}^{*}(x)
$$

- интерполяционный многочлен координатного множества В. Тогда функиия переноса в первый разряд для функции $F\left(x_{1}, \ldots, x_{k}\right)$ в координатном множестве В удовлетворяет соотношению

$$
\begin{aligned}
& \gamma_{1}^{B}\left(F\left(a_{1}, \ldots, a_{k}\right)\right) \equiv-F^{*}\left(a_{1}, \ldots, a_{k}\right)+\sum_{i=1}^{k} \frac{\partial F}{\partial x_{i}}\left(a_{1}, \ldots, a_{k}\right) \Psi_{B}^{*}\left(a_{i}\right) \\
&-\Psi_{B}^{*}\left(F\left(a_{1}, \ldots, a_{k}\right)\right) \quad(\bmod p), \quad a_{1}, \ldots, a_{k} \in B .
\end{aligned}
$$

Доказательство. Введем обозначение

$$
F\left(a_{1}, \ldots, a_{k}\right)=c
$$

По теореме 1 ,

$$
\gamma_{0}^{B}(c)=\Psi_{B}\left(c^{q^{n-1}}\right) \equiv \Psi_{B}\left(c^{q}\right)=c^{q}+p \Psi_{B}^{*}\left(c^{q}\right) \equiv c^{q}+p \Psi_{B}^{*}(c) \quad\left(\bmod p^{2}\right) .
$$

Отсюда

$$
p \gamma_{1}^{B}(c) \equiv c-\gamma_{0}^{B}(c) \equiv c-c^{q}-p \Psi_{B}^{*}(c) \quad\left(\bmod p^{2}\right), \quad c \in R
$$

Ввиду (7),

$$
c^{q}=F\left(a_{1}^{q}, \ldots, a_{k}^{q}\right)+p F^{*}\left(a_{1}, \ldots, a_{k}\right) .
$$

Следовательно,

$$
p \gamma_{1}^{B}(c) \equiv c-F\left(a_{1}^{q}, \ldots, a_{k}^{q}\right)-p F^{*}\left(a_{1}, \ldots, a_{k}\right)-p \Psi_{B}^{*}\left(F\left(a_{1}, \ldots, a_{k}\right)\right) \quad\left(\bmod p^{2}\right) .
$$


Для доказательства формулы (8) остается проверить, что

$$
F\left(a_{1}, \ldots, a_{k}\right)-F\left(a_{1}^{q}, \ldots, a_{k}^{q}\right) \equiv p \sum_{i=1}^{k} \frac{\partial F}{\partial x_{i}}\left(a_{1}, \ldots, a_{k}\right) \Psi_{B}^{*}\left(a_{i}\right) \quad\left(\bmod p^{2}\right) .
$$

Ввиду линейности частных производных, это соотношение достаточно доказать для случая, когда $F\left(a_{1}, \ldots, a_{k}\right)=x_{1}^{m_{1}} \cdots x_{k}^{m_{k}}-$ моном.

Так как $a_{i} \in B$, ввиду (9)

$$
a_{i}=\gamma_{0}^{B}\left(a_{i}\right) \equiv a_{i}^{q}+p \Psi_{b}^{*}\left(a_{i}\right) \quad\left(\bmod p^{2}\right) .
$$

Тогда

$$
\begin{aligned}
F\left(a_{1}, \ldots, a_{k}\right)-F\left(a_{1}^{q}, \ldots, a_{k}^{q}\right) & =a_{1}^{m_{1}} \cdots a_{k}^{m_{k}}-\left(a_{1}^{m_{1}} \cdots a_{k}^{m_{k}}\right)^{q} \\
& \equiv\left(a_{1}^{q}+p \Psi_{B}^{*}\left(a_{1}\right)\right)^{m_{1}} \cdots\left(a_{k}^{q}+p \Psi_{B}^{*}\left(a_{k}\right)\right)^{m_{k}}-\left(a_{1}^{m_{1}} \cdots a_{k}^{m_{k}}\right)^{q} \\
& \equiv p \sum_{i=1}^{k} a_{1}^{q m_{1}} \cdots a_{i-1}^{q m_{i-1}} m_{i} a_{i}^{q\left(m_{i}-1\right)} \Psi_{B}^{*}\left(a_{i}\right) a_{i+1}^{q m_{i}+1} \cdots a_{k}^{q m_{k}} \\
& \equiv p \sum_{i=1}^{k} a_{1}^{m_{1}} \cdots a_{i-1}^{m_{i}-1} m_{i} a_{i}^{m_{i}-1} \Psi_{B}^{*}\left(a_{i}\right) a_{i+1}^{m_{i}+1} \cdots a_{k}^{m_{k}} \\
& =p \sum_{i=1}^{k} \frac{\partial F}{\partial x_{i}}\left(a_{1}, \ldots, a_{k}\right) \Psi_{B}^{*}\left(a_{i}\right) \quad\left(\bmod p^{2}\right),
\end{aligned}
$$

что и требовалось доказать.

Следствие 4. Если $B=\Gamma(R)-$-адическое координатное множество, то

$$
\gamma_{1}\left(F\left(a_{1}, \ldots, a_{k}\right)\right)=-F^{*}\left(a_{1}, \ldots, a_{k}\right), \quad a_{1}, \ldots, a_{k} \in \Gamma(R) .
$$

Доказательство. Согласно следствию 1,

$$
\Psi_{\Gamma(R)}(x)=x
$$

Следовательно, $\Psi_{\Gamma(R)}^{*}(x)=0$, и в формуле $(8)$ остается только первое слагаемое.

Заметим, что первое слагаемое $-F^{*}\left(a_{1}, \ldots, a_{k}\right)$ в формуле (8) не зависит от координатного множества $B$. Ввиду (9),

$$
\gamma_{0}^{B}(c) \equiv \gamma_{0}(c)+p \Psi_{B}^{*}(c) \quad\left(\bmod p^{2}\right), \quad c \in R,
$$

поэтому значение $\Psi_{B}^{*}(c)$ можно расценивать как отклонение элемента $\gamma_{0}^{B}(c)$ от $\gamma_{0}(c)$. При этом сумма в формуле (8) есть производная функции $F\left(x_{1}, \ldots, x_{k}\right)$ в направлении вектора $\left(\Psi_{B}^{*}\left(a_{1}\right), \ldots, \Psi_{B}^{*}\left(a_{k}\right)\right)$.

Пользуясь теоремой 2, вычислим функции переноса в первый разряд в важных частных случаях - для операций сложения, умножения и вычисления линейной комбинации элементов $a_{1}, \ldots, a_{k}$. 
Предложение 4. Пусть $r=G R\left(q^{n}, p^{n}\right), r=q / p, B$ - координатное множество кольцза $R, a_{1}, \ldots, a_{k} \in B u c_{0}, c_{1}, \ldots, c_{k} \in R$. Тогда

$$
\begin{aligned}
\gamma_{1}^{B}\left(c_{0}+c_{1} a_{1}+\ldots+c_{k} a_{k}\right) \equiv \gamma_{1}\left(c_{0}\right) & +\sum_{i=1}^{k} \gamma_{1}\left(c_{i}\right) a_{i} \\
& +\sum_{\substack{j_{0}+j_{1}+\ldots+j_{k}=p \\
0 \leqslant j_{i}<p}} \frac{1}{j_{0} ! j_{1} ! \cdots j_{k} !} c_{0}^{r j_{0}}\left(c_{1} a_{1}\right)^{r j_{1}} \ldots\left(c_{k} a_{k}\right)^{r j_{k}} \\
& +\sum_{i=1}^{k} c_{i} \Psi_{B}^{*}\left(a_{i}\right)-\Psi_{B}^{*}\left(c_{0}+c_{1} a_{1}+\ldots+c_{k} a_{k}\right) \quad(\bmod p) .
\end{aligned}
$$

Доказательство. Применяя формулу (8) для многочлена

$$
F\left(x_{1}, \ldots, x_{k}\right)=c_{0}+c_{1} x_{1}+\ldots+c_{k} x_{k},
$$

видим, что последние два слагаемых в (8) совпадают с последними двумя слагаемыми в $(10)$, и остается вычислить $F^{*}\left(a_{1}, \ldots, a_{k}\right)$. Применяя полиномиальную формулу, получим, что

$$
\begin{aligned}
F\left(x_{1}, \ldots, x_{k}\right)^{q} & =\left(c_{0}+c_{1} x_{1}+\ldots+c_{k} x_{k}\right)^{q} \\
& =c_{0}^{q}+c_{1}^{q} x_{1}^{q}+\ldots+c_{k}^{q} x_{k}^{q} \\
& \quad+\sum_{\substack{j_{0}+j_{1}+\ldots+j_{k}=p \\
0 \leqslant j_{i}<p}} \frac{q !}{j_{0} ! j_{1} ! \cdots j_{k} !} c_{0}^{j_{0}}\left(c_{1} x_{1}\right)^{j_{1}} \cdots\left(c_{k} x_{k}\right)^{j_{k}} .
\end{aligned}
$$

В силу теоремы Люка (см. теорему 4.71 в [1] или лемму 1 в [5]), при условиях $j_{0}+j_{1}+\ldots+j_{k}=q, 0 \leqslant j_{1}, \ldots, j_{k}<q$, полиномиальный коэффициент $q ! /\left(j_{0} ! j_{1} ! \cdots j_{k} !\right)$ делится на $p$. При этом он не делится на $p^{2}$ тогда и только тогда, когда $j_{i}$ делится на $r=q / p$ при всех $i=0,1, \ldots, k$. Следовательно,

$$
\begin{aligned}
F\left(x_{1}, \ldots, x_{k}\right)^{q} \equiv c_{0}^{q}+c_{1}^{q} x_{1}^{q}+\ldots+c_{k}^{q} x_{k}^{q} \\
+\sum_{\substack{j_{0}+j_{1}+\ldots+j_{k}=q \\
0 \leqslant j_{i}<q}} \frac{q !}{\left(r j_{0}\right) !\left(r j_{1}\right) ! \cdots\left(r j_{k}\right) !} c_{0}^{r j_{0}}\left(c_{1} x_{1}\right)^{r j_{1}} \cdots\left(c_{k} x_{k}\right)^{r j_{k}} \quad\left(\bmod p^{2}\right) .
\end{aligned}
$$

По той же теореме Люка,

$$
\left(\begin{array}{l}
p a \\
p b
\end{array}\right) \equiv\left(\begin{array}{l}
a \\
b
\end{array}\right) \quad(\bmod p)
$$

и по теореме Вильсона

$$
(p-1) ! \equiv-1 \quad(\bmod p) .
$$

Поэтому

$$
\frac{q !}{\left(r j_{0}\right) !\left(r j_{1} !\right) \cdots\left(r j_{k} !\right)} \equiv \frac{p !}{j_{0} ! j_{1} ! \cdots j_{k} !} \equiv-\frac{p}{j_{0} ! j_{1} ! \cdots j_{k} !} \quad\left(\bmod p^{2}\right)
$$


В итоге

$$
\begin{aligned}
F\left(x_{1}, \ldots, x_{k}\right)^{q} & \equiv c_{0}^{q}+c_{1}^{q} x_{1}^{q}+\ldots+c_{k}^{q} x_{k}^{q} \\
& -p \sum_{\substack{j_{0}+j_{1}+\ldots+j_{k}=q \\
0 \leqslant j_{i}<q}} \frac{1}{j_{0} ! j_{1} ! \cdots j_{k} !} c_{0}^{r j_{0}}\left(c_{1} x_{1}\right)^{r j_{1}} \ldots\left(c_{k} x_{k}\right)^{r j_{k}} \quad\left(\bmod p^{2}\right) .
\end{aligned}
$$

С другой стороны,

$$
\begin{aligned}
F\left(x_{1}^{q}, \ldots, x_{k}^{q}\right) & =c_{0}+c_{1} x_{1}^{q}+\ldots+c_{k} x_{k}^{q} \\
& =c_{0}^{q}+c_{1}^{q} x_{1}^{q}+\ldots+c_{k}^{q} x_{k}^{q}+\left(c_{0}-c_{0}^{q}\right)+\left(c_{1}-c_{1}^{q}\right) x_{1}^{q}+\ldots+\left(c_{k}-c_{k}^{q}\right) x_{k}^{q} .
\end{aligned}
$$

Ввиду леммы 4 в [8],

$$
c^{q} \equiv \gamma_{0}(c) \quad\left(\bmod p^{2}\right)
$$

поэтому

$$
c-c^{q} \equiv p \gamma_{1}(c) \quad\left(\bmod p^{2}\right), \quad c \in R
$$

Следовательно,

$$
\begin{aligned}
F\left(x_{1}^{q}, \ldots, x_{k}^{q}\right) \equiv c_{0}^{q}+c_{1}^{q} x_{1}^{q}+\ldots & +c_{r}^{q} x_{k}^{q} \\
& +p \gamma\left(c_{0}\right)+p \gamma\left(c_{1}\right) x_{1}^{q}+\ldots+p \gamma_{1}\left(c_{k}\right) x_{k}^{q} \quad\left(\bmod p^{2}\right) .
\end{aligned}
$$

Теперь $F^{*}\left(x_{1}, \ldots, x_{k}\right)$ вычисляется по формуле (7):

$$
\begin{aligned}
F^{*}\left(x_{1}, \ldots, x_{k}\right) \equiv & -\gamma_{1}\left(c_{0}\right)-\sum_{i=1}^{k} \gamma_{1}\left(c_{i}\right) x_{i}^{q} \\
& \quad-\sum_{\substack{j_{0}+j_{1}+\ldots+j_{k}=p \\
0 \leqslant l_{i}<p}} \frac{1}{j_{0} ! j_{1} ! \cdots j_{k} !} c_{0}^{r j_{0}}\left(c_{1} x_{1}\right)^{r j_{1}} \cdots\left(c_{k} x_{k}\right)^{r j_{k}} \quad(\bmod p) .
\end{aligned}
$$

Подставляя выражение для $F^{*}$ в формулу (8) и учитывая, что

$$
a_{i}^{q} \equiv a_{i} \quad(\bmod p)
$$

получим соотношение (10).

Следствие 5. Пусть $a_{1}, \ldots, a_{k} \in B$, где $B$-координатное множество кольца $R$. Тогда

$$
\begin{aligned}
& \gamma_{1}^{B}\left(a_{1}+\ldots+a_{k}\right) \equiv \sum_{\substack{j_{1}+j_{2}+\ldots+j_{k}=p \\
0 \leqslant j_{i}<p}} \frac{1}{j_{1} ! j_{2} ! \cdots j_{k} !} a_{1}^{r j_{1}} \cdots a_{k}^{r j_{k}} \\
& +\sum_{i=1}^{k} \Psi_{B}^{*}\left(a_{i}\right)-\Psi_{B}^{*}\left(a_{1}+\ldots+a_{k}\right)(\bmod p) .
\end{aligned}
$$

В частности, если $a, b \in B$, то

$$
\gamma_{1}^{B}(a+b) \equiv \sum_{j=1}^{p-1} \frac{1}{j !(p-j) !} a^{r j} b^{r(p-j)}+\Psi_{B}^{*}(a)+\Psi_{B}^{*}(b)-\Psi_{B}^{*}(a+b) \quad(\bmod p) .
$$


Наиболее компактные формулы получаются в случае $p$-адического координатного множества.

Следствие 6. Если $a_{1}, \ldots, a_{k} \in \Gamma(R)$, mo

$$
\gamma_{1}\left(a_{1}+\ldots+a_{k}\right) \equiv \sum_{\substack{j_{1}+j_{2}+\ldots+j_{k}=p \\ 0 \leqslant j_{i}<p}} \frac{1}{j_{1} ! j_{2} ! \cdots j_{k} !} a_{1}^{r j_{1}} \cdots a_{k}^{r j_{k}}(\bmod p) .
$$

В частности, если $a, b \in \Gamma(R)$, то

$$
\gamma_{1}(a+b) \equiv \sum_{j=1}^{p-1} \frac{1}{j !(p-j) !} a^{r j} b^{r(p-j)} \quad(\bmod p) .
$$

Доказательство. Для доказательства первого следствия нужно положить $c_{0}=0$, $c_{1}=\ldots=c_{k}=1$ в предложении 4. Второе следствие получается, если учесть, что в случае $B=\Gamma(R)$ выполняется равенство $\Psi_{\Gamma(R)}^{*}=0$.

Отметим, что формула для $\gamma_{1}(a+b)$ в случае $p$-адического и $p$-ичного координатных множеств была получена ранее (см. лемму 2.2 в [4]).

Если $R=\mathbf{Z}_{p^{n}}$, то $q=p$ и в формулах предложения 4 и его следствий нужно положить $r=1$.

Предложение 5. Если $a_{1}, \ldots, a_{k} \in B, c \in R=G R\left(q^{n}, p^{n}\right)$, то

$$
\begin{aligned}
\gamma_{1}^{B}\left(c a_{1} \cdots a_{k}\right) \equiv \gamma_{1}(c) & a_{1} \cdots a_{k} \\
& +c \sum_{i=1}^{k} a_{1} \cdots a_{i-1} a_{i+1} \cdots a_{k} \Psi_{b}^{*}\left(a_{i}\right)-\Psi_{B}^{*}\left(c a_{1} \cdots a_{k}\right) \quad(\bmod p) .
\end{aligned}
$$

В частности, если $a, b \in B$, то

$$
\gamma_{1}^{B}(a b) \equiv a \Psi_{B}^{*}(b)+b \Psi_{B}^{*}(a)-\Psi_{B}^{*}(a b) \quad(\bmod p) .
$$

Доказательство. Пусть

$$
F\left(x_{1}, \ldots, x_{k}\right)=c x_{1} \cdots x_{k} .
$$

Тогда

$$
\begin{aligned}
F\left(x_{1}, \ldots, x_{k}\right)^{q}-F\left(x_{1}^{q}, \ldots, x_{k}^{q}\right) & =\left(c^{q}-c\right) x_{1}^{q} \cdots x_{k}^{q} \\
& \equiv\left(\gamma_{0}(c)-c\right) x_{1}^{q} \cdots x_{k}^{q} \\
& \equiv-p \gamma_{1}(c) x_{1}^{q} \cdots x_{k}^{q}\left(\bmod p^{2}\right) .
\end{aligned}
$$

Согласно (7),

$$
F^{*}\left(x_{1}, \ldots, x_{k}\right) \equiv-\gamma_{1}(c) x_{1}^{q} \cdots x_{k}^{q} \quad(\bmod p)
$$

при этом

$$
F^{*}\left(x_{1}, \ldots, x_{k}\right) \equiv-\gamma_{1}(c) a_{1} \cdots a_{k} \quad(\bmod p) .
$$

Подставляя выражение для $F^{*}$ в формулу (8), получаем требуемое соотношение.

Если в условиях предложения $5 B=\Gamma(R)$, мы получим равенство

$$
\bar{\gamma}_{1}(a b)=0 \text {. }
$$

Это согласуется с тем, что $p$-адическое координатное множество замкнуто относительно умножения. 


\section{4. Случай $p$-ичного координатного множества}

Особый интерес представляет $p$-ичное координатное множество, поскольку оно наиболее часто используется на практике, а также обладает интересными свойствами с теоретической точки зрения.

Согласно теоремам 1 и 2, описание нулевой и первой координатных функций в координатном множество $B$ сводится к изучению интерполяционного многочлена $\Psi_{B}(x)$. Оказывается, что для $p$-ичного координатного множества свойства интерполяционного многочлена оказываются связанными с понятием простого регулярного числа. Всюду далее в этом параграфе $R=\mathbf{Z}_{p^{n}}$ и $B=\{0,1, \ldots, p-1\}-p$-ичное координатное множество.

Пусть $p-$ простое нечетное число. Введем обозначение

$$
s_{k}(p)=1^{k}+2^{k}+\ldots+(p-1)^{k}, \quad k \geqslant 1 .
$$

Выполняются соотношения (приведенные далее факты можно найти в [2])

$$
p\left|s_{k}(p), \quad 1 \leqslant k \leqslant p-2 ; \quad p^{2} \nmid s_{1}(p) ; \quad p^{2}\right| s_{2 a+1}(p), \quad 3 \leqslant 2 a+1 \leqslant p-2 .
$$

Пара $(p, 2 a), 2 \leqslant 2 a \leqslant p-3$, называется иррегулярной, если $p^{2} \mid s_{2 a}(p)$, в противном случае эта пара называется регулярной. Число иррегулярных пар называется индексом иррегулярности числа $p$ и обозначается $i i(p)$. Например, пара $(37,32)$ иррегулярна, так как $s_{32}(37)$ делится на $37^{2}$, и можно проверить, что $i i(37)=1$. Если $i i(p)=0$, то простое число $p$ называется регулярным. Среди простых чисел $p<100$ нерегулярны только 39, 59 и 67. Согласно гипотезе Зигеля (см. [2], с. 489), доля простых регулярных чисел среди всех простых чисел составляет $1 / \sqrt{e} \approx 0,61$. В литературе принято другое определение регулярного простого числа - на языке числа классов дивизоров $p$-кругового поля, введенное Е. Куммером в середине XIX века в связи с доказательством великой теоремы Ферма. Куммер установил, что пара $(p, 2 a)$ регулярна тогда и только тогда, когда числитель числа Бернулли $B_{2 a}$ не делится на $p$. Последнее свойство равносильно данному нами определению (см. [2]).

Теорема 3. Пусть $B=\{0,1,2, \ldots, p-1\}-p$-ичное координатное множество кольцุа $R=\mathbf{Z}_{p^{n}}, p \geqslant 3$. Тогда коэффициенты интерполяциионного многочлена

$$
\Psi_{B}(x)=\psi_{p-1} x^{p-1}+\ldots+\psi_{1} x \in R[x]
$$

координатного множества В удовлетворяют соотношениям

$$
\begin{gathered}
\psi_{1} \equiv 1 \quad(\bmod p), \quad \psi_{3} \equiv \psi_{5} \equiv \ldots \equiv \psi_{p-2} \equiv 0 \quad(\bmod p), \\
\psi_{2}=\psi_{4}=\ldots=\psi_{p-3}=0, \quad \psi_{p-1}=\frac{1}{2} p,
\end{gathered}
$$

и если $2 \leqslant 2 a \leqslant p-3$, то $\psi_{p-2 a} \equiv 0\left(\bmod p^{2}\right)$ тогда и только тогда, когда $(p, 2 a)-$ иррегулярная пара.

Доказательство. По следствию 1 ,

$$
\Psi_{B}(x) \equiv x \quad(\bmod p) .
$$

Отсюда следуют соотношения (11). 
Занумеруем элементы координатных множеств $B$ и $\Gamma(R)$ в таком порядке, что $\beta_{t} \equiv t$ $(\bmod p), t=0,1, \ldots, p-1$. Тогда $\beta_{p-t}=-\beta_{t}, t=1,2, \ldots, p-1$, и из (5) получаем, что

$$
\begin{aligned}
\psi_{l} & =\frac{1}{p-1} \sum_{t=1}^{p-1} t \beta_{t}^{p-1-l}=\frac{1}{p-1} \sum_{t=1}^{r}\left(t \beta_{t}^{p-1-l}+(p-t) \beta_{p-t}^{p-1-l}\right) \\
& =\frac{1}{p-1} \sum_{t=1}^{r}\left(t \beta_{t}^{p-1-l}+(p-t)\left(-\beta_{t}\right)^{p-1-l}\right), \quad l \in\{2,3, \ldots, p-1\},
\end{aligned}
$$

где $r=(p-1) / 2$. Пусть $l \in\{2,3, \ldots p-1\}-$ четное число. Тогда

$$
\begin{aligned}
\psi_{l} & =\frac{1}{p-1} \sum_{t=1}^{r} p \beta_{t}^{p-1-l}=\frac{p}{2(p-1)} \sum_{t=1}^{r}\left(\beta_{t}^{p-1-l}+\beta_{p-t}^{p-1-l}\right) \\
& =\frac{p}{2(p-1)} \sum_{t=1}^{p-1} \beta_{t}^{p-1-l}=\frac{p}{2(p-1)} \sum_{t=1}^{p-1} \beta_{*}^{t(p-1-l)},
\end{aligned}
$$

где $\beta_{*}-$ произвольный образующий мультипликативной группы $\Gamma(R) \backslash 0$ порядка $p-1$. Вычисляя сумму геометрической прогрессии и учитывая равенство

$$
\beta_{*}^{p-1}=e,
$$

получаем соотношения (12).

В силу следствия 3 ,

$$
\psi_{l} \equiv \chi_{l} \quad\left(\bmod p^{2}\right), \quad l \in\{2,3, \ldots, p-1\} .
$$

По определению характеристического многочлена $\chi_{B}(x)$,

$$
\chi_{l}=(-1)^{p-l} \sigma_{p-l}(1,2, \ldots, p-1), \quad l \in\{1,2, \ldots, p-1\},
$$

где $\sigma_{r}(1,2, \ldots, p-1)$ - элементарная симметрическая функция степени $k$ от $p-1$ переменной. Воспользуемся формулами Ньютона, связывающими симметрические функции $s_{k}$ и $\sigma_{k}$ (см. [3], с. 264):

$$
s_{k}-s_{k-1} \sigma_{1}+s_{k-2} \sigma_{2}-\ldots+(-1)^{k-1} s_{1} \sigma_{k-1}+(-1)^{k} k \sigma_{k}=0, \quad k \in\{1,2, \ldots, p-1\} .
$$

Подставим сюда вместо $s_{j}, \sigma_{j}$ величины $s_{j}(p), \sigma_{j}(1,2, \ldots, p-1)$. Так как

$$
\sigma_{l}(1,2, \ldots, p-1) \equiv 0 \quad(\bmod p), \quad j \in\{1,2, \ldots, p-2\},
$$

получим, что

$$
s_{k}(p) \equiv 0 \quad(\bmod p), \quad k \in\{1,2, \ldots, p-2\} .
$$

Учитывая это сравнение и вновь используя формулы Ньютона, получим, что

$$
s_{k}(p) \equiv(-1)^{k+1} k \sigma_{k}(1,2, \ldots, p-1) \quad\left(\bmod p^{2}\right) .
$$

В итоге

$$
\begin{aligned}
\psi_{l} & \equiv \chi_{l}=(-1)^{p-1} \sigma_{p-l}(1,2, \ldots, p-1) \\
& \equiv(-1)^{p-l}(-1)^{p-l+1} \frac{1}{p-l} s_{p-l}(p) \\
& \equiv \frac{1}{l} s_{p-l}(p) \quad\left(\bmod p^{2}\right), \quad l \in\{2,3, \ldots, p-1\}
\end{aligned}
$$


Следовательно, $\psi_{p-2 a} \equiv 0\left(\bmod p^{2}\right)$ тогда и только тогда, когда $p^{2} \mid s_{2 a}(p)$, то есть $(p, 2 a)$ - иррегулярная пара.

Следствие 7. В условиях теоремы 3 соотношения $\psi_{3} \not \equiv 0\left(\bmod p^{2}\right), \psi_{5} \not \equiv 0\left(\bmod p^{2}\right)$, $\ldots, \psi_{p-2} \not \equiv 0\left(\bmod p^{2}\right)$ выполняются тогда и только тогда, когда $p-$ простое регулярное число.

В работах $[5,6]$ понятие простого регулярного числа появлялось при решении двух различных задач - при нахождении ранга $p$-ичного представления линейной рекуррентной последовательности (ЛРП) над полем $\mathbf{Z}_{p}$ и ранга первой координатной последовательности ЛРП максимального периода над кольцом $\mathbf{Z}_{p}$. Полученные выше результаты объясняют причину такого совпадения. Это связано не с какими-то общими свойствами ЛРП над полем $\mathbf{Z}_{p}$ и кольцом $\mathbf{Z}_{p^{n}}$, а с тем, что в обеих работах используется $p$-ичное координатное множество кольца $\mathbf{Z}_{p^{n}}$, свойства которого и связаны с регулярностью простого числа $p$.

Используем полученные результаты для уточнения вида функции переноса в первый разряд при сложении и умножении. Из следствия 5 следует, что если $a_{1}, \ldots, a_{k} \in B$, то

$$
\begin{aligned}
\gamma_{1}^{B}\left(a_{1}+\ldots+a_{k}\right) \equiv & \sum_{\substack{j_{1}+j_{2}+\ldots+j_{k}=p \\
0 \leqslant j_{i}<p}} \frac{1}{j_{1} ! j_{2} ! \cdots j_{k} !} a_{1}^{j_{1}} \cdots a_{k}^{j_{k}} \\
& +\sum_{l=1}^{p-1} \psi_{l}^{*}\left(a_{1}^{l}+\ldots+a_{k}^{l}-\left(a_{1}+\ldots+a_{k}\right)^{l}\right) \quad(\bmod p) .
\end{aligned}
$$

Согласно теореме 3, последнее равенство можно уточнить:

$$
\begin{aligned}
& \gamma_{1}^{B}\left(a_{1}+\ldots+a_{k}\right) \equiv \sum_{\substack{j_{1}+j_{2}+\ldots+j_{k}=p \\
0 \leqslant j_{i}<p}} \frac{1}{j_{1} ! j_{2} ! \cdots j_{k} !} a_{1}^{j_{1}} \cdots a_{k}^{j_{k}} \\
& +\frac{1}{2}\left(a_{1}^{p-1}+\ldots+a_{k}^{p-1}-\left(a_{1}+\ldots+a_{k}\right)^{p-1}\right) \\
& +\sum_{\substack{2 \leqslant 2 a \leqslant p-3 \\
(p, 2 a) \text { есть регулярная пара }}} \psi_{p-2 a}^{*}\left(a_{1}^{p-2 a}+\ldots+a_{k}^{p-2 a}\right.
\end{aligned}
$$

причем в последней сумме коэффициент $\psi_{p-2 a}^{*}$ при каждом слагаемом не сравним с нулем по модулю $p$. Аналогично, по предложению 5

$$
\gamma_{1}^{B}\left(a_{1} \cdots a_{k}\right) \equiv \sum_{i=1}^{k} a_{1} \cdots a_{i-1} a_{i+1} \cdots a_{k} \Psi_{B}^{*}\left(a_{i}\right)-\Psi_{B}^{*}\left(a_{1}, \ldots, a_{k}\right) \quad(\bmod p) .
$$

Отсюда с учетом теоремы 3 получаем, что

$$
\begin{aligned}
& \gamma_{1}^{B}\left(a_{1} \cdots a_{k}\right) \equiv \frac{1}{2} a_{1} \cdots a_{k}\left(a_{1}^{p-2}+\ldots+a_{k}^{p-2}-\left(a_{1} \cdots a_{k}\right)^{p-2}\right) \\
&+\sum_{\substack{2 \leqslant 2 a \leqslant p-3 \\
(p, 2 a) \text { есть регуляная пара }}} \psi_{p-2 a}^{*} a_{1} \cdots a_{k}\left(a_{1}^{p-2 a-1}+\ldots+a_{k}^{p-2 a-1}\right. \\
&-\left(a_{1} \ldots a_{k}\right)^{p-2 a-1}(\bmod p) .
\end{aligned}
$$


В частности, если $p$ - регулярное простое число, то все пары $(p, 2 a)$ регулярны, откуда следует, что

$$
\begin{aligned}
\gamma_{1}^{B}\left(a_{1}+\ldots+a_{k}\right) \equiv & \sum_{\substack{j_{1}+j_{2}+\ldots+j_{k} \\
0 \leqslant j_{i}<p}} \frac{1}{j_{1} ! j_{2} ! \cdots j_{k} !} a_{1}^{j_{1}} \ldots a_{k}^{j_{k}} \\
& +\frac{1}{2}\left(a_{1}^{p-1}+\ldots+a_{k}^{p-1}-\left(a_{1}+\ldots+a_{k}\right)^{p-1}\right) \\
& +\sum_{\substack{3 \leqslant l \leqslant p-2 \\
l \text { нечетно }}} \psi_{l}^{*}\left(a_{1}^{l}+\ldots+a_{k}^{l}-\left(a_{1}+\ldots+a_{k}\right)^{l}\right) \quad(\bmod p)
\end{aligned}
$$

И

$$
\begin{aligned}
\gamma_{1}^{B}\left(a_{1} \cdots a_{k}\right) & \equiv \frac{1}{2} a_{1} \cdots a_{k}\left(a_{1}^{p-2}+\ldots+a_{k}^{p-2}-\left(a_{1} \cdots a_{k}\right)^{p-2}\right) \\
& +\sum_{\substack{3 \leqslant l \leqslant p-2 \\
l}} \psi_{l}^{*} a_{1} \cdots a_{k}\left(a_{1}^{l-1}+\ldots+a_{k}^{l-1}-\left(a_{1} \cdots a_{k}\right)^{l-1}\right) \quad(\bmod p) .
\end{aligned}
$$

Формулы (13) и (15) уточняют лемму 2.2 работы [4].

При использовании формул (13) и (14) оказывается полезной таблица иррегулярных пар для простых чисел $p<8000$, которую можно найти в [2]. Для нахождения коэффициентов $\psi_{l}^{*}$ проще всего использовать предложение 2 и его следствие.

Рассмотрим пример. Пусть $p=r$ и $a, b \in\{0,1,2,3,4\}$. Тогда

$$
a+b=a \oplus b+p \gamma_{1}^{B}(a+b)
$$

где

$$
a \oplus b=\gamma_{0}^{B}(a+b)=a+b \quad(\bmod 5)
$$

Рассмотрим характеристический многочлен

$$
\begin{aligned}
\chi_{b}(x) & =x(x-1)(x-2)(x-3)(x-4) \\
& =x^{5}-10 x^{4}+35 x^{3}-50 x^{2}+24 x \\
& =x^{5}-x+5\left(-2 x^{4}+7 x^{3}-10 x^{2}+5 x\right) .
\end{aligned}
$$

Отсюда

$$
\chi_{b}^{*}(x)=-2 x^{4}+7 x^{3}-10 x^{2}+5 x
$$

По предложению 2,

$$
\Psi_{B}^{*}(x) \equiv \chi_{B}^{*}(x) \quad(\bmod p),
$$

поэтому

$$
\Psi_{B}^{*}(x) \equiv 3 x^{4}+2 x^{3} \quad(\bmod 5)
$$


Так как простое число 5 регулярно, то функция переноса в первый разряд $\gamma_{1}^{B}(a+b)$ выписывается непосредственно по формуле (15):

$$
\begin{aligned}
\gamma_{1}^{B}(a+b) \equiv & \sum_{j=1}^{4} \frac{1}{j !(p-j) !} a^{j} b^{p-j}+\frac{1}{2}\left(a^{4}+b^{4}-(a+b)^{4}\right)+2\left(a^{3}+b^{3}-(a+b)^{3}\right) \\
\equiv & 4 a b^{4}+3 a^{2} b^{3}+3 a^{3} b^{2}+4 a^{4} b+3 a b^{3} \\
& \quad+2 a^{2} b^{2}+3 a^{3} b+4 a b^{2}+4 a^{2} b \quad(\bmod 5) .
\end{aligned}
$$

Аналогично,

$$
a b=a \otimes b+p \gamma_{1}^{B}(a b)
$$

где

$$
a \otimes b=\gamma_{0}^{B}(a b)=a b \quad(\bmod 5),
$$

а функция $\gamma_{1}^{B}(a b)$ выписывается по формуле (16):

$$
\gamma_{1}^{B}(a b) \equiv 3\left(a b^{4}+a^{4} b-a^{4} b^{4}\right)+2\left(a b^{3}+a^{3} b-a^{3} b^{3}\right) \quad(\bmod 5) .
$$

\section{Список литературы}

1. Берлекэмп Э., Алгебраическая теория кодирования. Мир, Москва, 1971.

2. Боревич 3. И., Шафаревич И. Р., Теория чисел. Наука, Москва, 1985.

3. Кострикин А. И., Введение в алгебру. Наука, Москва, 1977.

4. Кузьмин А. С., Нечаев А. А., Линейные рекуррентные последовательности над кольцами Галуа. Алгебра и логика (1995) 4, №4, 96-116.

5. Куракин В. Л., Представления над кольцом $\mathbf{Z}_{p^{n}}$ линейной рекуррентной последовательности максимального периода над полем $G F(p)$. Дискретная математика (1992) 4, №4, 96-116.

6. Куракин В. Л., Первая координатная последовательность линейной рекурренты максимального периода над кольцом Галуа. Дискретная математика (1994) 6, №2, 88-100.

7. Нечаев А. А., Конечные кольца главных идеалов. Матем. сборник (1973) 91, №3, 350-336.

8. Нечаев А. А., Код Кердока в циклической форме. Дискретная математика (1989) 1, №4, 123139.

9. Kurakin V. L., Kuzmin A. S., Mikhalev A. V., Nechaev A. A., Linear recurring sequences over rings and modules. J. Math. Sci. (1995) 76, №6, 2793-2915.

10. McDonald B. R., Finite rings with identity. Marcel Dekker, New York, 1974.

11. Nechaev A. A., Finite rings with applications. In: Handbook of algebra, 5 (Hazewinkel M., ed.). Elsevier, Amsterdam, 2008, pp. 217-320.

Статья поступила 1.02.2010. 